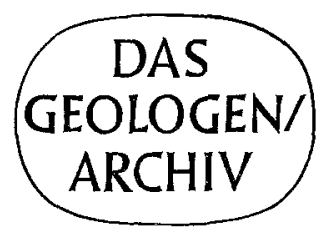

B E R L I N $/ S C H O C N E$ B E R G A $M$ M P A R K 11

\author{
DEUTSCHE GEOLOGEN IN NORDAFRIKA
}

\title{
Wilhelm Schimper
}

Der erste Deutsche, der in Nordafrika geologisch gearbeitet hat, war wohl WilHelm SoHIMPeR (1804 bis 1878), KarI SoHimpers jüngerer Bruder. Über sein abenteuerliches Leben lese man in der Allgemeinen Deutschen Biographie 31, $279 \mathrm{ff}$. nach. Auf seinen Reisen nach Abessinien fand er in Adoa beim König von Tigré Schutz und Aufnahme. Er wurde Statthalter der Provinz Antitscho und heiratete eine Abessinierin. Außer mit botanischen und zoologischen Forschungen beschäftigten ihn auch geologische Sammlungen und die Bearbeitung einer geologischen Karte des Landes, die wohl die erste geologische Karte eines nordafrikanischen Gebiets war. Seine erste geologische Publikation ist von 1848: „Constitution geologique de quelques parties de l'Abessynie et observations relatives à la météorologie ${ }^{\text {“ }}$ (Compt.rend. XXVI). 1877 erschien in Bd.12 der Zeitschrift der Gesellschaft für Erdkunde in Berlin „Geologie von Arrho, Abyssinien“. Schrmpers geologische Karte wurde wohl nie gedruckt.

E. H.

Das geologische Material Max BLANokenhorns an Handschriften, Tagebüchern, Kartierungsblättern usw. wird dankenswerterweise dem GA überwiesen werden. Aus dem reichen Bestand an Briefen ScHweINfurths an BLANCKENHORN drucken wir folgenden Brief (im GA) ab:

\section{G. Sehweinfurth an M. Blanckenhorn}

\section{Lieber Freund,}

(Palermo, Ende Febr. 1906)

Ihr Brief vom 19. Febr. $1906 \mathrm{kam}$ heute an und bereitete große Freude. Ich danke Thnen bestens für die ausführlichen und so wichtigen Nachrichten. Thre Bedenken wegen der Terrassen werden nun gewiß nachdem Sie das Land verlassen vollständig gehoben sein. Das wichtigste für mich war:

1. die Absage von der lacustrinen Idee (bez. des älteren Diluviums);

2. das Vorhandensein des Nils zur Zeit der Pluvialepoche. Hoffentlich werden Sie beides in Shaghab bestätigt finden. Ich fürchte nur, daß Sie Schwierigkeiten gefunden haben werden das große Uadi Girrua zu besuchen, denn der Eshet el Wuz ist doch nur ein Vorwerk.

$\mathrm{Ob}$ noch eine selbständige Mittelterrasse vorhanden sei werden Sie schließlich auch ins Reine gebracht haben. Mein großer Wunsch wäre nur, daß Sie wirkliche Palaeolithica in den Schichten mit Unio finden möchten; denn bei Schelluit, im Unterlauf des Rinnsals sind doch keine vorhanden und „Strepyien“ ist doch noch Iange kein „Chelleen“, bezw. Moustierien. Sie vergaßen Ihre Ansicht hinsichtlich der $10 \mathrm{~m}$ hohen Steilwand von Nilerde am 